\title{
DNA Analysis Based on the Local Structural Disruption to the Duplexes Carrying a Luminous Lanthanide Complex
}

\author{
Toshihiro Ihara, ${ }^{\dagger}$ Yusuke KITAMura, Yusuke TsuJIMura, and Akinori Jyo \\ Department of Applied Chemistry and Biochemistry, Graduate School of Science and Technology, \\ Kumamoto University, 2-39-1 Kurokami, Kumamoto 860-8555, Japan
}

\begin{abstract}
The two DNA conjugates (split probes) carrying a metal chelator form an integrated luminous lanthanide $\left(\operatorname{Ln}^{3+}: \mathrm{Tb}^{3+} \mathrm{or}\right.$ $\mathrm{Eu}^{3+}$ ) complex on the complementary template DNA (target). The luminous property of this $\mathrm{Ln}^{3+}$ complex has been used for DNA assay. The intensity of the luminescence was affected by the local structural disruption caused by one-base mispairing around the complex. Among the mispairings systematically introduced around the $\operatorname{Ln}^{3+}$ center, vicinal mispairings to the center decreased the emission intensity more. This would be a novel nucleobase-discriminating principle, in which the split probes bind the target tightly, yet still retain sequence selectivity.
\end{abstract}

(Received March 12, 2011; Accepted April 19, 2011; Published June 10, 2011)

\section{Introduction}

We have been engaged in the development of methods for gene detection through cooperative recognition of the target. ${ }^{1-13}$ In a series of studies, we showed that the two ODN (oligo deoxyribonucleic acid) conjugates bearing a metal chelating group on their terminus cooperatively hybridize to the adjacent sequences on the target in the presence of a half mole of specific metal ions. ${ }^{14}$ We now present the techniques of split probes based on the complexation between a luminous lanthanide ion $\left(\mathrm{Ln}^{3+}: \mathrm{Tb}^{3+}\right.$ or $\left.\mathrm{Eu}^{3+}\right)$ and the two ODN conjugates carrying a metal chelator. EDTA (ethylenediaminetetraacetic acid) and phen (1,10-phenanthroline) were covalently attached to the ODNs to prepare a pair of the ODN conjugate as the split probes. When the ternary duplex consisting of the target and the two conjugates forms, as shown in Fig. 1(a), the EDTA and phen moieties of the conjugates face each other to form an integrated cavity for $\mathrm{Ln}^{3+}$ accommodation. The two units are expected to function as dehydration/capturing and sensitizer units for $\mathrm{Ln}^{3+}$, respectively. The stability constants of phen- $\mathrm{Ln}^{3+}$ are not high. Therefore, without the guide by the template (target), phen could not coordinate to $\mathrm{Ln}^{3+}$ under the experimental conditions. That is, the luminous $\mathrm{Ln}^{3+}$ complexes form only when the targets are added into the solution.

We previously demonstrated the benefits of this approach for the analysis of the thiopurine S-methyltransferase (TPMT) gene. ${ }^{15,16}$ The luminescence from $\mathrm{Ln}^{3+}$ complexes formed in the tandem duplexes consisting of 7 mer EDTA-ODN conjugate, 20 mer phen-ODN conjugate, and the target highly depended on the single base displacements on a 7 mer half of the target. The difference in thermal stability of the duplexes caused by a mispairing was involved to discriminate the SNPs (single

\footnotetext{
† To whom correspondence should be addressed.

E-mail: toshi@chem.kumamoto-u.ac.jp

This paper is dedicated to the memory of Prof. Makoto Takagi, an outstanding teacher and researcher.
}

nucleotide polymorphisms); the 7 mer probe does not bind to the target with a single base displacement under the assay conditions. In this system, therefore, the probe ODNs should be adequately short to attain a higher selectivity, because the negative impact to the thermal stability that comes from one base mismatch (destabilization caused by a mispairing) is larger for shorter duplexes. Short probes, however, would not work in diluted solution because of their lower binding affinity to the targets. Therefore, the SNP discrimination principle itself should be modified to achieve both high fidelity and high binding affinity. ${ }^{17,18}$ Here we used a pair of the longer conjugates (15 mer) as the split probes to ensure higher affinity. The sequences of the conjugates were designed so that the bases of mispairings were located around the end where the EDTA or phen was tethered. We hypothesized that the local structural disruption on the duplexes caused by a mispairing around the metal center affects the luminescence intensity. ${ }^{17}$ In this report, we examined the effect of the position of the mispairing on the luminescence from $\mathrm{Tb}^{3+}$ and $\mathrm{Eu}^{3+}$ complexes cooperatively formed in the tandem duplexes consisting of the two $15 \mathrm{mer}$ conjugates and the 30 mer targets.

\section{Experimental}

\section{General equipment and materials}

ODNs were purchased from Japan BioServices (Japan) and Hokkaido System Science (Japan), or synthesized on an Expedite 8900 (Applied Biosystems) DNA synthesizer with dimethoxytrityl phosphoramidite reagents purchased from Proligo (USA) and Glen Research (USA). All other reagents were purchased and used without further purification. The ODNs or ODN conjugates (probes) were purified from the crude mixture by RP-HPLC (reversed phase-HPLC, LC-2000 plus inert system, Jasco) equipped with an ODS column (octadecylsilyl, Wakopak ${ }^{\circledR}$-Wakosil-II 5C18 RS, 4.6 mm $(\phi) \times 150 \mathrm{~mm}(\mathrm{w})$, Wako). ${ }^{19}$ UV spectral data were acquired on a UV-1650PC (Shimadzu) or V-560 (JASCO). MALDI-TOF 
(a)

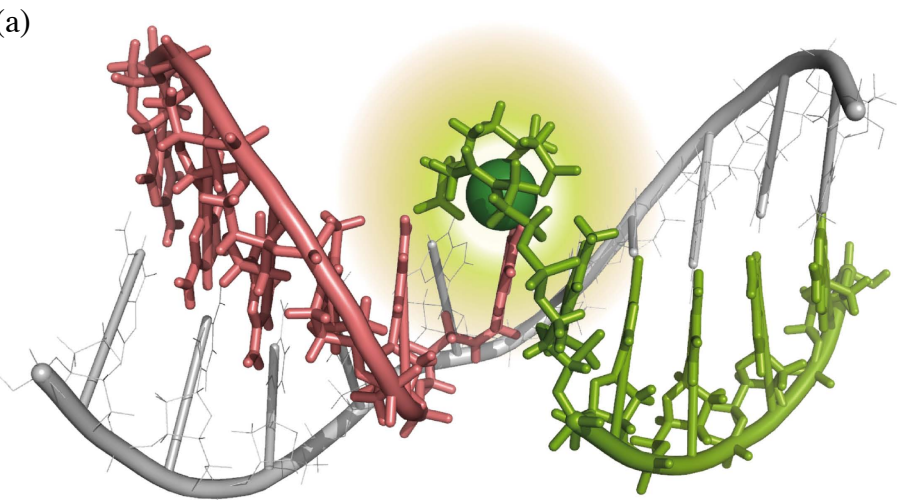

(b)

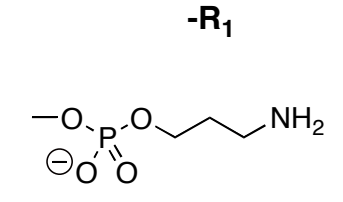

5'amino15<smiles>COP(=O)([O-])OCCCNC(=O)c1cc2cccnc2c2ncccc12</smiles>

Phen-15<smiles>COP(=O)(O)OCCCNC(=O)CN(CCN(CC(=O)O)CC(=O)O)CC(=O)O</smiles>

$-\mathbf{R}_{2}$

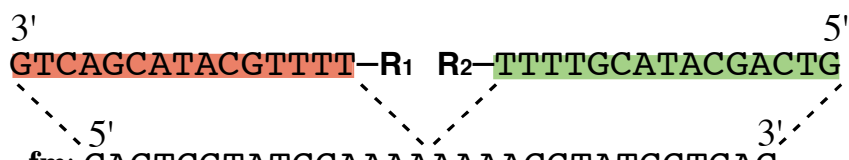

fm: CAGTCGTATGCAAAAAAAACGTATGCTGAC

mm(-3N): CAGTCGTATGCANAAAAAACGTATGCTGAC

mm(-2N): CAGTCGTATGCAANAAAAACGTATGCTGAC

mm(-1N): CAGTCGTATGCAAANAAAACGTATGCTGAC

mm(+1N): CAGTCGTATGCAAAANAAACGTATGCTGAC

mm(+2N): CAGTCGTATGCAAAAANAACGTATGCTGAC

mm(+3N): CAGTCGTATGCAAAAAANACGTATGCTGAC

$$
\mathbf{N}=\mathrm{G}, \mathrm{C} \text {, or } \mathrm{T}
$$

Fig. 1 Design of the system. (a) One of the possible structures of $\mathrm{Ln}^{3+}$ complex formed in ternary tandem duplex. The model was geometry-optimized by AMBER* force field with GB/SA (generalized Born/surface area) solvent model using MacroModel Ver. 9.1. Red strand (left), a part of Phen-15; green strand (right), a part of EDTA-15; gray strand, a part of $\mathbf{f m}$; sphere (center), $\mathrm{Ln}^{3+}$. (b) The structures and sequences of the split probes (DNA conjugates: Phen-15 and EDTA-15) and the fullmatch $(\mathbf{f m})$ and mismatch targets $(\mathbf{m m}(\mathbf{n N})(\mathbf{n}=-3,-2,-1,+1,+2$, or $+3, \mathbf{N}=\mathrm{G}, \mathrm{C}$, or T)).

MS (matrix-assisted laser desorption ionization mass spectrometry) of ODNs and ODN conjugates were performed on an Autoflex III (Bruker Daltonics) in negative ion mode.

Synthesis of the split probes (ODN conjugates)

The conjugates Phen-15 and EDTA-15 (Fig. 1(b)) were synthesized by the coupling of primary amino groups of $5^{\prime}$ amino15 and $\mathbf{3}^{\prime}$ 'amino15 with $N$-hydroxysuccinimide ester of 5-carboxy-1,10-phenanthroline and dianhydride of EDTA, respectively, purified by RP-HPLC, and subsequently identified with MALDI-TOF MS using 3-hydroxypicolinic acid (3-HPA) as matrix. Full procedures of the synthesis of the two ODN conjugates are shown in our previous reports. ${ }^{16}$

\section{UV melting}

Thermal denaturation experiments were carried out in phosphate buffer solution (10 mM, pH 7.0) containing $1.0 \mathrm{M}$ $\mathrm{NaCl}$ on a UV-1650PC (Shimadzu) UV/vis spectrophotometer equipped with a Peltier thermal controller. The concentrations of each component of the ternary duplexes and $\mathrm{Ln}^{3+}$ were all $1 \mu \mathrm{M}$. Prior to the beginning of each melt, the samples were degassed at $85^{\circ} \mathrm{C}$ for $5 \mathrm{~min}$ and then annealed with slow cooling 
to $0^{\circ} \mathrm{C}$. In the denaturation experiments, the solutions were heated at a rate of $0.5 \mathrm{deg} \mathrm{min}^{-1}$ after equilibration for $30 \mathrm{~min}$ at $0^{\circ} \mathrm{C}$. The melting profiles were monitored by the absorption change at $260 \mathrm{~nm}$ while blowing dry $\mathrm{N}_{2}$ gas (only below room temperature) over the cuvette to prevent dew condensation. The temperature that gave the maximum first derivative of the melting curve, $T_{\max }$, was used as a measure of the thermal stability of the duplexes. Thermal denaturation experiments were repeated $2-3$ times for each sample and the reported $T_{\max }$ values are the average of all duplicates or triplicates for a given sample.

The molar extinction coefficients of the targets, $\mathbf{f m}$ and $\mathbf{m m}(\mathbf{n N})$ s, were calculated using those of the nearest-neighbor dinucleoside monophosphates at $260 \mathrm{~nm}$ as reported in the literature. ${ }^{20}$ The concentrations (extinction coefficients) of the conjugates, Phen-15 and EDTA-15, were determined as shown in our previous reports. ${ }^{16}$

\section{Luminescence measurements}

Luminescence spectra were measured using an LS-55 luminescence spectrometer (Perkin-Elmer) equipped with a Peltier thermal controller. The sample space was purged with $\mathrm{N}_{2}$ to prevent moisture condensation on the cell when measuring below room temperature. Time-resolved emission spectra were obtained for $2 \mathrm{~ms}$ gate time after $50 \mu$ s delay using a pulsed xenon source (excitation at $280 \mathrm{~nm}$ ) in the buffer solution (10 mM HEPES containing 1.0 M NaCl, pH 7.0).

Luminescence intensities were also measured using the plate reader, ARVO MX 1420 Multilabel Counter (Perkin-Elmer). The signals were collected for $2 \mathrm{~ms}$ gate time after $50 \mu$ s delay using a pulsed xenon source (excitation at $280 \mathrm{~nm}$ ) through band-pass filters of $545 \mathrm{~nm}$ (7 nm bandwidth) and $615 \mathrm{~nm}$ (8.5 $\mathrm{nm}$ bandwidth) for $\mathrm{Tb}^{3+}$ and $\mathrm{Eu}^{3+}$ systems, respectively. Samples were dissolved in $100 \mu \mathrm{L}$ of buffer solutions $(10 \mathrm{mM}$ HEPES containing 1.0 M NaCl, $\mathrm{pH} 7.0$ ), dispensed in a 96-well plate, and subjected to measurement.

\section{Results and Discussion}

Design and preparation of the system

It is not difficult to attain sequence selective recognition using adequately short probes. However, the thermal stability of the short probes is not high. Such low stability would make it difficult to apply the probes for dilute analyte solutions. Here, a set of the long ODN conjugates carrying a metal chelator, EDTA and phen, were synthesized as capture and sensitizer probes for $\mathrm{Ln}^{3+}$ (Fig. 1(b)). The 15 mer sequences of the two probes were designed so as to form a tandem duplex with a target, in which their auxiliary units face each other, providing a microenvironment to accommodate $\mathrm{Ln}^{3+}$. To verify the effect of the local structural disruption caused by a mispairing on the luminescence intensity, we prepared a series of the model targets. Besides the target with fully complementary sequence (fm), the 18 target ODNs containing one base displacement $(\mathbf{m m}(\mathbf{n N}), \mathbf{n}=-3,-2,-1,+1,+2$, or $+3 ; \mathbf{N}=\mathrm{G}, \mathrm{C}$, or T) were used for luminescence measurements. Mismatched bases were systematically introduced to $\mathbf{m m}(\mathbf{n N})$ at between \pm 3 nucleobases around the opposite point where two probes meet. ${ }^{17}$

The coupling reaction yields for preparing the conjugates were $>80 \%$, which were calculated roughly from the comparisons of integrated peak area between the unreacted amino-ODNs (5'amino15 and $\mathbf{3}$ 'amino15) and the products (Phen-15 and EDTA-15). Both of the conjugates were identified with MALDI-TOF MS (calcd for [Phen-15-H] ${ }^{-}$4882.15, found 4881.28; calcd for [EDTA-15-H]- 4984.31, found 4983.15).
Table 1 Melting temperatures $\left(T_{\max }\right)$ of the tandem duplexes in the absence and presence of $\operatorname{Ln}^{3+}$

\begin{tabular}{lccc}
\hline \multirow{2}{*}{ Target } & None & \multicolumn{2}{c}{$\mathrm{Ln}^{3+}$} \\
\cline { 3 - 4 } & & $\mathrm{Eu}^{3+}$ & $\mathrm{Tb}^{3+}$ \\
\hline $\mathbf{f m}$ & 59.2 & 62.9 & 63.0 \\
$\mathbf{m m}(\mathbf{n N})$ & $56.8-58.9^{\mathrm{a}}$ & $58.4-62.8^{\mathrm{a}}$ & $59.1-62.5^{\mathrm{a}}$ \\
\hline
\end{tabular}

a. The ranges of $T_{\max }$ of 18 tandem duplexes with $\mathbf{m m}(\mathbf{n N}) \mathrm{s}$ are shown.

(a)

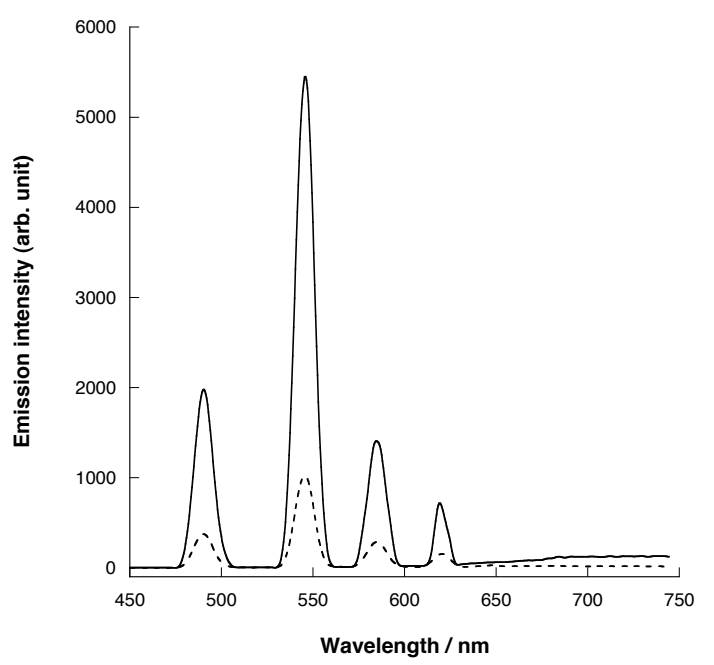

(b)

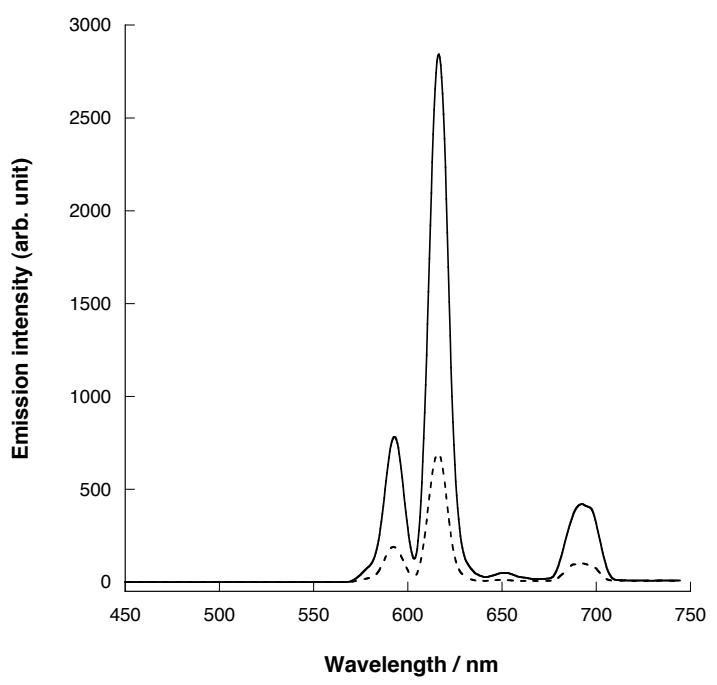

Fig. 2 Time-resolved emission $\left(\lambda_{\text {ex }}=280 \mathrm{~nm}\right)$ spectra of the solutions containing Phen-15, EDTA-15, and the targets in the presence of (a) $\mathrm{Tb}^{3+}$ or (b) $\mathrm{Eu}^{3+}$. The concentrations of all DNAs and $\mathrm{Ln}^{3+}$ were $1 \mu \mathrm{M}$. Solid and dashed curves show the spectra for the duplexes with $\mathbf{f m}$ and $\mathbf{m m}(\mathbf{- 1 T})$, respectively. The spectra were obtained with $2 \mathrm{~ms}$ gate time after $50 \mu$ s delay using a pulsed xenon source in the buffer solution (10 mM HEPES containing $1.0 \mathrm{M} \mathrm{NaCl}$, $\mathrm{pH} 7.0)$

\section{Thermal stability}

Before conducting the luminescence measurements, we subjected all of the tandem duplexes consisting of EDTA-15, Phen-15, and fm or $\mathbf{m m}(\mathbf{n N})$ to UV melting experiments in the 
(a)
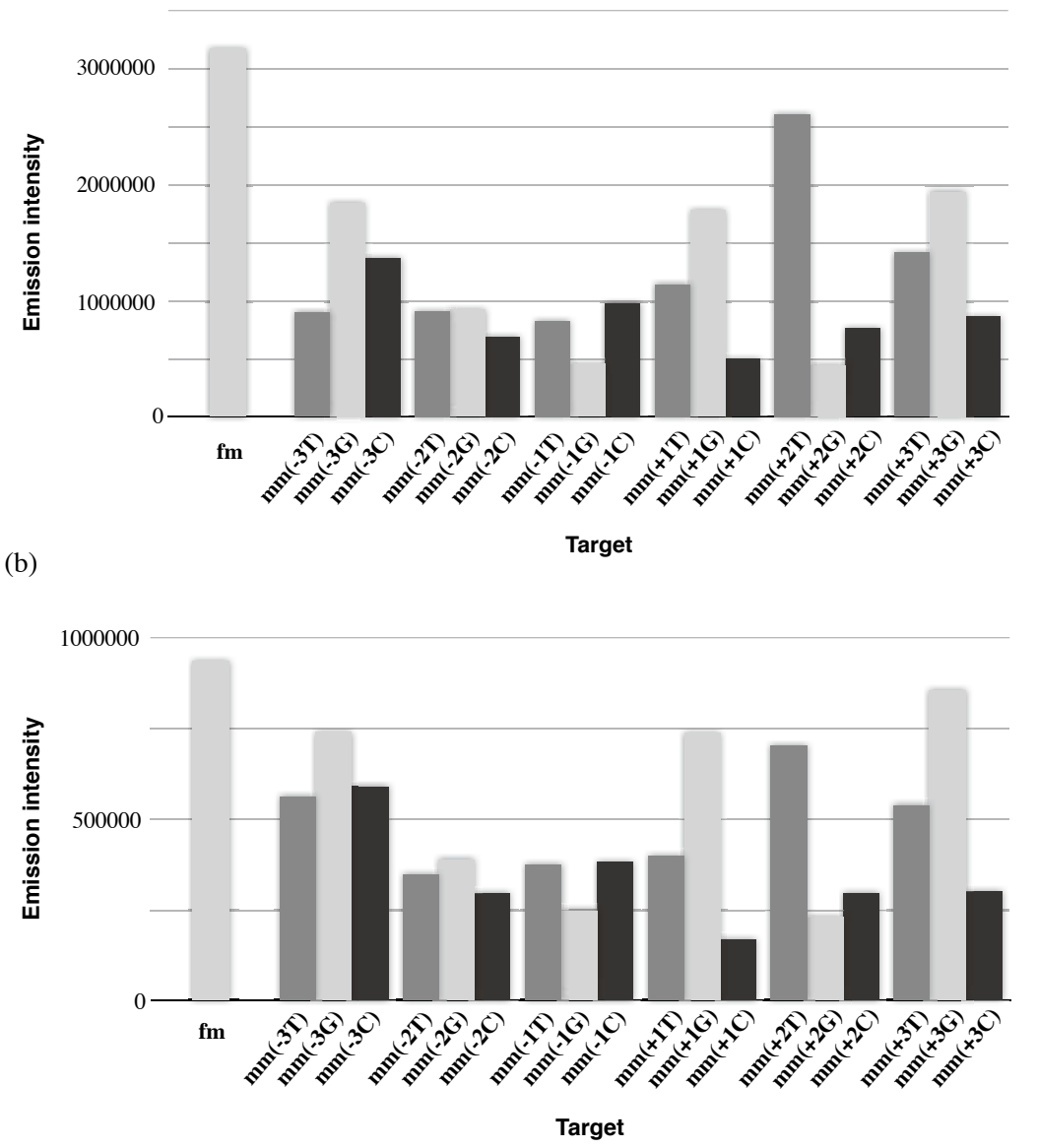

Fig. 3 High-throughput luminescence measurements using a plate reader. The luminescence intensities were measured by time-resolved mode for all tandem duplexes $(1 \mu \mathrm{M}$ each of the DNA components and $\mathrm{Ln}^{3+}$ ). Signals were collected for $2 \mathrm{~ms}$ gate time after $50 \mu$ s delay using a pulsed xenon source (excitation at $280 \mathrm{~nm}$ ) through band-pass filters of $545 \mathrm{~nm}(7 \mathrm{~nm}$ bandwidth) and $615 \mathrm{~nm}$ (8.5 nm bandwidth) for (a) $\mathrm{Tb}^{3+}$ and (b) Eu ${ }^{3+}$ systems, respectively. Samples were dissolved in $100 \mu \mathrm{L}$ of buffer solutions (10 mM HEPES containing $1.0 \mathrm{M} \mathrm{NaCl}, \mathrm{pH}$ 7.0), dispensed in a 96-well plate, and subjected to measurement.

presence and absence of $\mathrm{Ln}^{3+}$. Changes of the absorbance at $260 \mathrm{~nm}$ versus temperature gave typical sigmoidal melting curves for all duplexes. Here we used a pair of the conjugates that have sequences symmetric to each other. Therefore, the two conjugates were expected to dissociate cooperatively, because the thermal stabilities of the two successive duplexes are close to each other. Apparent $T_{\max }$ values for the duplexes estimated from the melting curves are summarized in Table 1. Both lanthanide ions seem to slightly stabilize the tandem duplex (fm/EDTA-15/Phen-15). We previously reported that little stabilization was observed for the control tandem duplex with unmodified ODN. ${ }^{16}$ Therefore, lanthanide ions seem to form ternary complexes with EDTA and phen at the site where the two probes meet.

While mispairings caused a reduction in duplex stabilities to some extent, all $T_{\max } \mathrm{s}$ were around $60^{\circ} \mathrm{C}$. Generally, the mispairings at inner positions (larger $\mathbf{n}$ in $\mathbf{m m}(\mathbf{n N}),+3$ or -3 ) of the duplex affect its thermal stability more. However, such a typical tendency could not be observed here for the series of the duplexes with $\mathbf{m m}(\mathbf{n N}) \mathrm{s}$ in the presence of $\mathrm{Ln}^{3+}$. Although the effect on the thermal stability of the duplex should be trivial for the mispairing itself at outer position $(\mathbf{n}=+1$ or -1$)$, the mispairing at +1 or -1 may disturb the $\mathrm{Ln}^{3+}$ complex formation to offset its positive contribution to the thermal stability of the duplex. Both effects (general dependence of mispairing position $v s$. perturbation of $\mathrm{Ln}^{3+}$ complex) would be closely connected and make the situation intricate. It might support our motivation of present research. The luminescence of the ternary complexes was measured sufficiently below the $T_{\max } \mathrm{s}$, because we want to know the effect of local structural disruption of the duplexes on the luminescence intensities.

Effect of mispairings in tandem duplexes on their luminescence Preliminary studies on luminescence properties of $\mathrm{Ln}^{3+}$-containing tandem duplexes were performed using a luminescence spectrometer prior to high throughput measurements using a plate reader. Time-resolved luminescence spectra were measured at $10^{\circ} \mathrm{C}$ intervals between 0 and $80^{\circ} \mathrm{C}$ for one fullmatched and 18 mismatched tandem duplexes in the presence of equimolar $\mathrm{Tb}^{3+}$ or $\mathrm{Eu}^{3+}$. Among them, the spectra of the duplexes fm/EDTA-15/Phen-15 and mm(-1T)/EDTA15/Phen-15 obtained at $0^{\circ} \mathrm{C}$ are shown in Fig. 2. Unique shapes of the emission spectra for $\mathrm{Ln}^{3+}$ complexes were observed for both $\mathrm{Tb}^{3+}$ and $\mathrm{Eu}^{3+}$ especially at lower temperatures for all of the 
duplexes. ${ }^{21} \quad$ As we expected, the fullmatched duplexes (fm/EDTA-15/Phen-15) provided the emission with highest intensities. Relative quantum yields for the emission from the $\mathrm{Ln}^{3+}$ complexes formed on fm/EDTA-15/Phen-15 were calculated to be 12 and $5.3 \%$ for $\mathrm{Tb}^{3+}$ and $\mathrm{Eu}^{3+}$, respectively, based on the value of $\left[\mathrm{Ru}(\mathrm{bpy})_{3}\right]^{2+}$ in aerated water $(\phi=0.028) .^{22}$ They are reasonable values for the $\mathrm{Ln}^{3+}$ complexes consisting of different two ligands in aqueous media. The lifetimes $\left(\tau_{\mathrm{Ln}^{3+}}\right)$ were estimated to be 1.26 and $1.34 \mathrm{~ms}$ for $\mathrm{Tb}^{3+}$ and $\mathrm{Eu}^{3+}$, respectively, which are typical of values found for $\mathrm{Ln}^{3+}$ complexes and are long enough to be applied to time-gating techniques.

All the duplexes were subjected to luminescence measurements using the plate reader under the conditions optimized by preliminary studies using the spectrometer mentioned above. Figure 3 shows the emission signal intensities observed for all duplexes at $25^{\circ} \mathrm{C}$. The signal intensity was affected by one-base displacements in a position dependent manner. Among the mispairings at $\mathbf{n}=-3,-2,-1,+1,+2$, and +3 , generally, central positions seemed to affect the emission intensity more. All of the targets (fm and $\mathbf{m m}(\mathbf{n N})$ s) form stable duplexes at $25^{\circ} \mathrm{C}$ regardless of the position and kinds of the mismatches. Therefore, the difference in the emission intensities observed here should be attributed to the difference in the extent of local structural disruption in the duplexes. The results showed the reasonable general tendency. Overall, the mismatches closer to the modified ends $(-1$ and +1$)$ of the conjugates affect the structure around $\mathrm{Ln}^{3+}$ complexes more than those of inner positions $(-3$ and +3$)$ in both conjugates. The signal contrasts between fm/EDTA-15/Phen-15 and mm(-1N)/EDTA-15/ Phen-15 were 4-7. The contrast is large enough to be recognized by one's naked eyes especially for the $\mathrm{Tb}^{3+}$ complex as shown in Fig. 4(a). Each mispairing (T-T, T-G, and T-C) seems to have its own position-dependent profile of emission intensity, at least in the sequence used in this study. These profiles were almost reproducible irrespective of the instruments, spectrometer or plate reader. Therefore, the unique emission profiles would be due to the propagation of certain local structural deviation or dynamic conformational fluctuation of the each mispairing to the moiety of $\mathrm{Ln}^{3+}$ complex. Although some of them might be based on the specific static structures such as T-G wobble base pair, ${ }^{23}$ it is difficult to explain or predict the profile, because the neighboring bases should somewhat affect the extent of propagation of the deviation to $\mathrm{Ln}^{3+}$ complex moiety. Anyway, as we expected, the results showed that the long probes could be used for SNP analysis with the discrimination principle based on the local structural disruption around the signaling moiety. Long DNA probes have some merits comparing with the short ones. They would be applicable to highly diluted solution thanks to their strong binding affinity. In addition to this, generally, a long probe can target on a unique sequence even from an enormously long DNA sample like a whole genome. Assuming the perfectly unbiased distribution of the four nucleobases (of course, it is not true in reality), a certain 16 mer tract can be a unique sequence in 3 billion-long human genome $\left(4^{16}>3 \times 10^{9}\right)$, while a certain 7 mer sequence would appear every $c a .20$ thousand-long sequences ( $c a .200$ thousand times appearance in a 3 billion-long sequence).

While the luminescence intensities measured at $0^{\circ} \mathrm{C}$ (data not shown) were more than those obtained at $25^{\circ} \mathrm{C}$ for all tandem duplexes, the overall profiles of position dependence and, therefore, the signal contrasts of any $\mathbf{~ m m ( n N ) / E D T A - 1 5 / P h e n - 1 5 ~}$ to fm/EDTA-15/Phen-15 were almost the same for both temperatures. That is, the difference in the emission intensities (a)

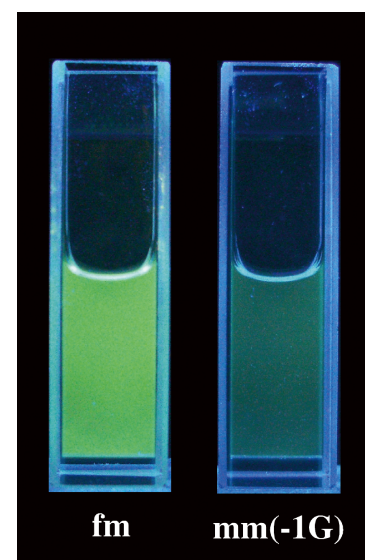

(b)

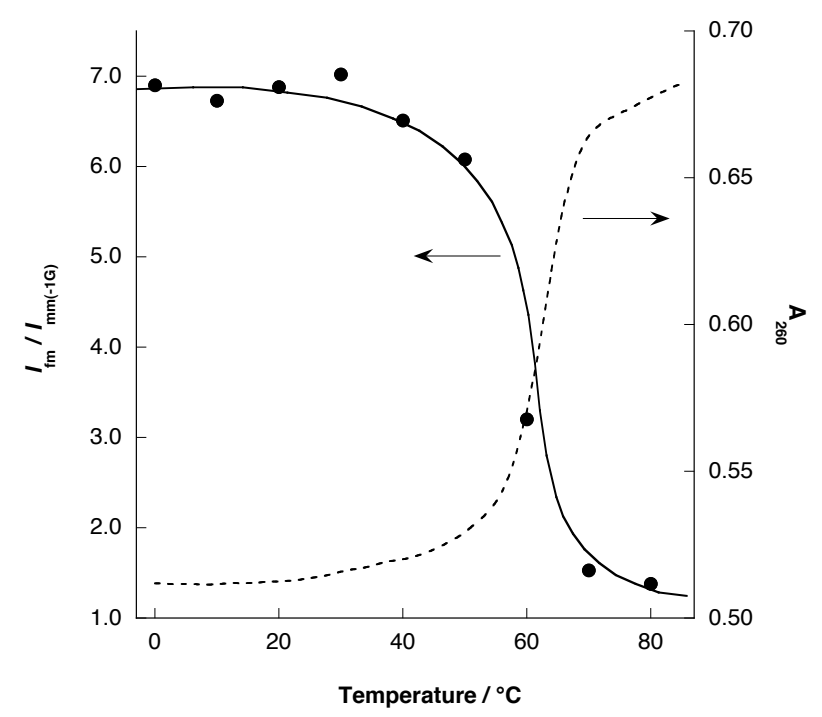

Fig. 4 Signal contrast between the duplexes with $\mathbf{~ m}$ and $\mathbf{~ m m}(\mathbf{- 1 G})$. (a) Luminescence images of the solutions of tandem duplexes with $\mathbf{f m}$ (left) and $\mathbf{m m}(-\mathbf{1 G})$ (right) in the presence of $\mathrm{Tb}^{3+}$ at $5^{\circ} \mathrm{C}(1 \mu \mathrm{M}$ for each of the components in the same buffer solution with Figs. 2 and 3). Excitation source: low-pressure mercury lamp $(6 \mathrm{~W})$. (b) The temperature dependence of the signal contrast between the two solutions, $I_{\mathrm{fm}} / I_{\mathrm{mm}(-1 \mathrm{G})}$ (closed circle) and the UV melting curve of fm/EDTA-15/Phen-15 in the presence of $\mathrm{Tb}^{3+}$ (broken curve) obtained under the same conditions.

between the two temperatures would be attributed to the intrinsic temperature-dependent properties of the emission from $\mathrm{Ln}(\mathrm{III})$ complexes. ${ }^{24}$ Actually, temperature dependence of signal contrasts seemed to coincide with the reversed shape of melting profile (Fig. 4(b)). It means that the present method would be applicable in wide range of temperature as long as it is lower than that of the duplex melting, because the discrimination principle does not rely on the subtle difference in thermal stability of the duplexes with the probes but on the local structural disruption caused by a mispairing. Therefore, we do not need to control the temperature strictly for SNP assay. This would be a great advantage in the assay for multiple targets.

For applying to practical highly sensitive assays, we have to improve the system regarding two points, 1) intrinsic intensity and 2) contrast $(\mathbf{f m} / \mathbf{m m}(\mathbf{n N}))$ of the luminescence signal from $\mathrm{Ln}^{3+}$ complexes. Exploring or developing the better sensitizers 
than phen should resolve the former issue. The latter would be improved by tuning the structure of the probes (DNA conjugates), such as, the length and rigidity of the linker chain.

\section{Conclusions}

We showed that the luminescence signal from $\mathrm{Ln}^{3+}$ complexes consisting of the two 15 mer probes on the targets is affected by the local structural disruption resulting from a mispairing in the duplexes. The dilemma in the length of DNA probes mentioned above would be avoided by using this feature as a discrimination principle. In addition to this, the system could be operated at a constant temperature regardless of the sequences of the targets as long as it is lower than the melting temperature of the duplexes.

\section{Acknowledgements}

The authors thank Prof. Sueda (Kyushu Institute of Technology) for his help in time-resolved luminescence assay using the plate reader. This work was partially supported by Priority Areas (Coordination Programming) (area 2107) from MEXT Japan and Grant-in-Aid for Scientific Research (B) (20350038) from JSPS (T. I.).

\section{References}

1. T. Ihara and M. Mukae, Anal. Sci., 2007, 23, 625.

2. D. M. Kolpashchikov, Chem. Rev., 2010, 110, 4709.

3. A. P. Silverman and E. T. Kool, Chem. Rev., 2006, 106, 3775 .

4. T. Ihara, A. Uemura, A. Futamura, M. Shimizu, N. Baba, S. Nishizawa, N. Teramae, and A. Jyo, J. Am. Chem. Soc., 2009, 131, 1386.

5. M. Mukae, T. Ihara, M. Tabara, and A. Jyo, Org. Biomol.
Chem., 2009, 7, 1349.

6. T. Ihara, T. Fujii, M. Mukae, Y. Kitamura, and A. Jyo, J. Am. Chem. Soc., 2004, 126, 8880.

7. Y. Kitamura, T. Ihara, K. Okada, Y. Tsujimura, Y. Shirasaka, M. Tazaki, and A. Jyo, Chem. Commun., 2005, 4523.

8. P. L. Paris, J. M. Langenhan, and E. T. Kool, Nucleic Acids Res., 1998, 26, 3789.

9. D. M. Kolpashchikov, J. Am. Chem. Soc., 2005, 127, 12442.

10. T. Endoh, M. Mie, H. Funabashi, T. Sawasaki, Y. Endo, and E. Kobatake, Bioconjugate Chem., 2007, 18, 956.

11. T. N. Grossmann, L. Röglin, and O. Seitz, Angew. Chem., Int. Ed., 2008, 47, 7119.

12. R. M. Franzini and E. T. Kool, J. Am. Chem. Soc., 2009, $131,16021$.

13. S. Ogasawara and K. Fujimoto, Angew. Chem., Int. Ed., 2006, 45, 4512.

14. T. Ihara, Y. Takeda, and A. Jyo, J. Am. Chem. Soc., 2001, 123, 1772.

15. Y. Kitamura, T. Ihara, Y. Tsujimura, Y. Osawa, M. Tazaki, and A. Jyo, Anal. Biochem., 2006, 359, 259.

16. Y. Kitamura, T. Ihara, Y. Tsujimura, Y. Osawa, D. Sasahara, M. Yamamoto, K. Okada, M. Tazaki, and A. Jyo, J. Inorg. Biochem., 2008, 102, 1921.

17. P. Arslan, T. Ihara, M. Mukae, and A. Jyo, Anal. Sci., 2008, 24, 173.

18. P. Arslan, A. Jyo, and T. Ihara, Org. Biomol. Chem., 2010, 8, 4843.

19. S. Agrawal, "Protocols for Oligonucleotide Conjugates", ed. S. Agrawal, 1994, Humana Press, Totowa.

20. C. R. Cantor and M. M. Warshaw, Biopolymers, 1970, 9, 1059.

21. I. Hemmilä and V. Laitala, J. Fluoresc., 2005, 15, 529.

22. K. Nakamura, Bull. Chem. Soc. Jpn., 1982, 55, 2697.

23. M. F. Goodman, Proc. Natl. Acad. Sci. U. S. A., 1997, 94, 10493.

24. Y. Hasegawa, Y. Wada, and S. Yanagida, J. Photochem. Photobiol., C, 2004, 5, 183. 